\title{
Preventing hepatitis B infection
}

Hepatitis B infection is endemic world-wide, with about 350 million people infected with the hepatitis B virus (HBV). HBV causes acute and chronic liver disease. After acute infection 5 to $10 \%$ fail to clear the virus and develop chronic liver disease or become healthy carriers. Chronic infection could lead to chronic hepatitis, cirrhosis and hepatocellular carcinoma. Chronic HBV is the ninth leading cause of death world-wide. HBV is transmitted parenterally, sexually and vertically. It can also be transmitted horizontally among young children. However, the method of transmission is often unclear. HBV infection is a major health problem in the SAARC region. Compared with countries such as India, Pakistan and Bangladesh, Sri Lanka has low rates of HBV infection in the general population. We have high prevalence rates among certain patient groups, eg. dialysis and kidney transplant patients, and thalassaemics who have had multiple blood transfusions.

Over the past several years travel within the SAARC region has increased. Stringent preventive measures will have to be taken if we are to keep the prevalence of HBV infection low. Preventive strategies include health education, using only disposable syringes and needles in clinics and hospitals, screening and testing of blood donors, and vaccination. Mass vaccination of newborns - inclusion of HBV vaccination in the expanded program of immunisation - is practiced in many developed countries. Even countries with a low prevalence (eg. USA) have adopted a policy of universal vaccination after it was found that uptake of vaccines was generally low in many of the groups at highest risk, and that about one-third of HBV infections had no identifiable source.

Sri Lanka is eligible to receive GAVI (Global Alliance for Vaccination and Immunisation) funding for hepatitis B vaccination for all infants at 2, 4 and 6 months. This program will commence in the year 2003 with 5 years' vaccine demand being donated by GAVI. By the year 2008 the Ministry of Health will be equipped to fund this program by adding on the sixth EPI vaccine. Hepatitis B vaccination (3 doses) provides over $90 \%$ protection as determined by antibody response. To date there are no data to support the need for booster doses of hepatitis B vaccine for at least 10 to 15 years in immunocompetent individuals who have responded to a primary course.

The Hepatitis B Awareness Week was launched for the first time in October 2000, in collaboration with party organisations world-wide, to address lack of awareness and to motivate the public. The campaign will start again in October 2001 to build on the achievements made last year. This year the campaign will run for a week, and will be called the Hepatitis B Action Week 2001. The primary objective of this year's campaign is to motivate people to take action to protect themselves and their families from hepatitis B. It will be supported at an international level by the American Liver Foundation and the Hepatitis B Foundation.

The Hepatitis Study Group, Colombo: for Hepatitis B Action Week 2001. 